\title{
CALCIUM CEVITAMATE IN THE TREATMENT OF ACUTE RHINITIS
}

\author{
SIMON L. RUSKIN, M.D.
}

\section{NEW YORK}

The discovery and synthesis of vitamin $\mathrm{C}$ has made possible a significant advance in calcium therapy sufficient to warrant reexamination of the value of calcium therapy in otolaryngology.

Vitamin $\mathrm{C}$ possesses the unique property of solubilizing and ionizing calcium to a degree not previously attainable by oral or intramuscular administration. In calcium cevitamate, the calcium salt of vitamin $\mathrm{C}$, there is available a more effective approach to calcium therapy than that of the previously used gluconate.

A comparison of the two products showed the following:

TABL.K 1

\begin{tabular}{|c|c|c|c|c|c|c|}
\hline Salt & $\begin{array}{l}\text { Per Cent } \\
\text { Solution } \\
\text { hy Weight }\end{array}$ & $\begin{array}{c}\text { Concen- } \\
\text { tration } \\
\text { Moles } \\
\text { Liter } \\
\end{array}$ & $\begin{array}{c}\text { Temp. } \\
\text { Drop } \\
\text { rC }\end{array}$ & $\begin{array}{l}\text { Per Cent } \\
\text { Ionization }\end{array}$ & $\begin{array}{c}\text { Yield Ca } \\
\text { lon per } \\
\text { Gram } \\
\text { Salt } \\
\end{array}$ & $\begin{array}{c}\text { Yield Ca } \\
\text { Jon Per } \\
\text { cc. Solu- } \\
\text { tion } \\
\end{array}$ \\
\hline Cul. Cevitamate & $5 \%$ & .123 & .577 & 74.6 & $.07(n)$ & .0037 \\
\hline Cal. Covitamate & $10 \%$ & .261 & 1.085 & 60.0 & .0963 & .0065 \\
\hline Cal. Cevitamati' & $20^{\circ}$ & .589 & 2.362 & 58.1 & $.01+5$ & .0137 \\
\hline Cal. Cevicamate & $30 \%$ & 1.008 & 3.940 & 55.3 & .0528 & .0226 \\
\hline Neo-Gluconate & $10 \%$ & .125 & .565 & 47.8 & $.03 \geq 3$ & $.00+8$ \\
\hline Neo-Giuconate & $20 \%$ & .273 & 1.126 & $+0 .{ }^{3}$ & .0273 & .0088 \\
\hline Calgulucon & $3 \%$ & .072 & .263 & 48.6 & $.0+52$ & .01014 \\
\hline
\end{tabular}

"Since the structure of the neo-calgluconate is not known, and since its empirical formula is $\left(\mathrm{C}_{i: 4} \mathrm{H}_{1:}, \mathrm{O}_{::,}\right) \mathrm{Ca}_{2,}$, we have assumed that it ionizes as follow's:

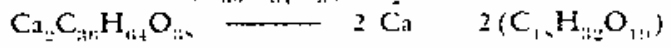

An analysis of these findings would tend to indicate the calcium cevitamate in 5 per cent solution was approximately equivalent to the neogluconate in 10 per cent solution and that the 10 per cent calcium cevitimate was approximately equivalent to the 20 per cent neogluconate.

The comparative series was run only to 30 per cent calicum cevitamate because the neogluconate solutions do not exceed 20 per cent. In considering the calglucon tablet which is only 3 per cent soluble the yield of calcium ions per cc. of solution was .0014, 
whereas that of the calcium cevitamate in only 30 per cent solution was .0226 , or about twenty times greater. If calculated on the basis of 100 per cent it would be in the vicinity of sixty times greater in calcium ion content per cc. solution. How important this factor can be in the question of calcium absorbability becomes apparent from the volume of solute necessary to obtain an equal amount of available calcium. For every $100 \mathrm{cc}$. of solute required for the absorption of calcium cevitamate, it would require $6,000 \mathrm{cc}$. of solute for an equal availability of calcium in calcium gluconate. Since the total fluid content of the gastro-intestinal canal is about 5,000 cc. and absorption of calcium occurs only in the upper intestine, the difference in absorbability between calcium cevitamate and calcium gluconate becomes apparent.

The action of cevitamic acid on the ionization of calicum opens up a field both of theory and practice. A comparison of the physiologic action of cevitamic acid and calcium shows an almost completely parallel action in bone metabolism, hemorrhagic diathesis, cell membrane permeability and detoxicating action. So close is this resemblance that one can interchange their functions. It is this element which suggests that cevitamic acid may be the factor which acts as the vehicle for the diffusible fraction of the serum calcium. There is a strong probability that the parathyroid hormone and cevitamic acid jointly balance the small fraction of ionized diffusible calicum. Greenwald is quoted by Cantarow as suggesting that seme of the calcium is bound to an organic substance in a citrate-like combination, the compound being intimately connected with the parathyroid hormone, probably diffusible and slightly ionized. Belief in the existence of such a compound is also shared by Klinke and by Sendray and Hastings. The latter drew attention to the similarity of action of the parathyroid hormone and citrate solutions in holding calcium in solution. In view of the fact that calcium citrate is only 0.8 per cent soluble, it is difficult to conceive of citrate as a calcium solubilizing agent. Cevitamic acid, however, has precisely the desired action en the ionization of calcium and its distribution in the intestinal canal, pituitary and adrenal makes it a much better hypothetical agent than citrate.

From the practical aspect, it is important to note that the injection of calcium cevitamate is nonirritating and better tolerated than gluconate.

In considering the action of calcium cevitimate in the series of cases here reported, one must take cognizance of the vitamin $\mathrm{C}$ action and its time-honored use as citric drinks in the treatment of the com- 
mon cold. However, if my theory as to the function of cevitamic acid is correct, one must attribute the ultimate benefit to calcium action.

A series of clinical trials were undertaken. All acute upper respiratory tract infections that applied for treatment in the ambulatory state and were free from fever, were considered in the group of the common cold.

Some were associated with acute exacerbations of chronic previously treated sinusitis, frequently of intluenzal character; others were of the simple infectious type associated with obvious exposure to persons suffering from cold.

The character of the response to treatment with calcium cevitamate is strikingly shown in the accompanying chart. The cases relieved by one injection were considered well in 24 hours or given a second injection on the second or third day. Cases associated with sinusitis received a continuation of the injections for one or two weeks because of the marked beneficial effects of the calcium cevitamate. Patients were advised to appear for treatment as close to the onset of the cold as possible, and were instructed to be mindful of the beginning dryness and discomfort of the nasopharynx and a fresh post-nasal discharge. These symptoms were observed to precede the coryza by about 12 to 24 hours. In a series of over two thousand injections, there were no complications incident to the injection. With but few exceptions there was no pain or reaction. There were, however, some instances of pain such as are incident to the injection of $3 \mathrm{cc}$. of any fluid, and soreness on pressure for a few days. This latter condition varied with the location of the injection, being absent if placed deep, slightly above the upper outer quadrant of the gluteus. Although a much larger series cf injections was given than are here recorded, only cases of upper respiratory conditions affecting the nose and ear are presented. The period of study covered two years and included a hundred cases. Some of the patients returned promptly for injections at the onset of or during a cold, several times during the year. In the acute cold, the injections were given daily in alternating sides, or every other day for one to four injections. In cases associated with sinusitis, two injections were given for the period of the treatment. Allergic patients were given two injections weekly, supplemented by 10 to 15 grains of nucleic acid three times daily. Improvement was noted as moderate, marked or complete. It would seem an extravagant statement to say that all cases showed improvement, yet with but a few isolated exceptions, all benefited by the reduction in nasal congestion and discharge and 
rapidity of recovery. The usual period of exhaustion following a cold was remarkably shortened.

The accompanying list of case reports represents 100 cases in continuous series treated with calcium cevitamate. In no case was there any complication associated with the injection. There were no sloughs, and only one case showed, on repeated injection, a tendency to lumpiness which gradually disappeared, thus establishing the nonirritating character of the solution and its ready absorption. Although in this series of cases 296 injections were administered intramuscularly, over 2,000 injections of the calcium cevitamate have been administered without any complications.

The cases reported comprise those cf upper respiratory tract infection, of which 48 were the common cold, 38 were combined acute rhinitis and sinusitis and two acute laryngitis, 12 cases had nasal allergy. The tabulation of results showed 42 per cent completely relieved, as a rule after the first or second injection, comprising chiefly the cases of acute cold, 35 per cent of which were markedly improved comprised chiefly the cases of acute cold combined with sinusitis, and 3 per cent but moderately improved comprised the more or less chronic types. The allergic cases responded remarkably well.

In presenting this work on clinical material, one is mindful of the fact that animal experimentation would be valuable. However, since the etiologic agent of the common cold has not yet been isolated, there remains no other control than clinical application which in this study gave conclusive and gratifying results.

\section{SUMMARY}

1. The value of calcium therapy in otolaryngology has been enhanced through the use of the calcium salt of vitamin $\mathrm{C}$, the calcium cevitamate.

2. A comparative test of the physical properties of calcium cevitamate and calcium gluconate shows a marked difference in solubility and ionization.

3. Since an ampule of $3 \mathrm{cc}$. of a 15 per cent solution of calcium cevitamate was injected, each dose represented about $450 \mathrm{mgs}$. of vitamin C. The calcium content is about 11 per cent when calculated as calcium oxide.

4. Calcium cevitamate would appear to be practically an abortive in the treatment of the common cold.

351 WEST 86TH STREET. 
TABLF: 11

\begin{tabular}{|c|c|c|c|c|c|}
\hline Cance $\#$ & Patienc & Sex & Date & Diagnosis & Comment \\
\hline 1 & B. B. & $M$ & 324,1937 & Acute cold & $\begin{array}{l}\text { Mirked improve- } \\
\text { ment }\end{array}$ \\
\hline 2 & c. $G$. & $\mathrm{F}$ & $+5,1937$ & Acute cold & Complete relief \\
\hline 3 & E. K. & F & $+9,1937$ & Acute cold & Complete relief \\
\hline 4 & J.G. & $\mathrm{F}$ & 45,1937 & Acute cold & Complete relict \\
\hline 5 & I. G. & F: & $+5,1937$ & $\begin{array}{l}\text { Acute cold } \\
\text { Rt. ethmoiditis }\end{array}$ & $\begin{array}{l}\text { Marked improve- } \\
\text { ment }\end{array}$ \\
\hline 6 & H.S. & $\mathrm{F}$ & $+1,2,5,7,1937$ & $\begin{array}{l}\text { Grippe, Icft } \\
\text { Sphenoiditis }\end{array}$ & $\begin{array}{l}\text { Marked improve- } \\
\text { ment }\end{array}$ \\
\hline 7 & P. D. & $\mathbf{M}$ & $+6,1937$ & $\begin{array}{l}\text { Aphthous stom- } \\
\text { atitis. Acute } \\
\text { cold }\end{array}$ & $\begin{array}{l}\text { Complete relief } \\
\text { jtomatitis disap- } \\
\text { peared afteer one } \\
\text { injection }\end{array}$ \\
\hline B & G. B. & $\mathbf{M}$ & $4 \cdot 7,1937$ & Acute laryngitis & $\begin{array}{l}\text { Marked improve } \\
\text { ment }\end{array}$ \\
\hline 9 & L. G. & F & $+7,12,1937$ & Acute cold & Complete relief \\
\hline 10 & D. S. & F & $\ldots \ldots$ & $\begin{array}{l}\text { Bilateral maxif- } \\
\text { lary and eth- } \\
\text { moidal sinusitis }\end{array}$ & $\begin{array}{l}\text { Improvern'r noted } \\
\text { with cach injec- } \\
\text { tion }\end{array}$ \\
\hline \pm 1 & l. $\mathrm{K}$. & $M$ & $119,11,1936$ & $\begin{array}{l}\text { Acute rhinitis } \\
\text { Bilateral eth- } \\
\text { moiditis }\end{array}$ & $\begin{array}{l}\text { Marked impruve- } \\
\text { ment }\end{array}$ \\
\hline 12 & P.S. & $\xi$ & $\begin{array}{rl}11 & 10,15,127,1936 \\
3 & 31,1937\end{array}$ & $\begin{array}{l}\text { Bilaterial eth- } \\
\text { moiditis }\end{array}$ & $\begin{array}{l}\text { Marked improve- } \\
\text { mene }\end{array}$ \\
\hline $1 ;$ & W. H. & M & +20.1937 & Acute cold & Complete relief \\
\hline If & R, R. & $\mathrm{F}$ & $+27,1937$ & $\begin{array}{l}\text { Bilateral eth- } \\
\text { moiditis }\end{array}$ & $\begin{array}{l}\text { Moderate im- } \\
\text { provement }\end{array}$ \\
\hline 15 & F. G. & $\mathrm{F}$ & +27.1937 & $\begin{array}{l}\text { Bilateral cth- } \\
\text { moiditis }\end{array}$ & $\begin{array}{l}\text { Marked improve- } \\
\text { ment }\end{array}$ \\
\hline 16 & D. D. D. & $M$ & 428,1937 & Acute rhinitis & $\begin{array}{l}\text { Miarked improve- } \\
\text { ment }\end{array}$ \\
\hline 17 & M. B. & $\mathrm{F}$ & $53,7,1937$ & $\begin{array}{l}\text { Bibateral eth- } \\
\text { mosiditis }\end{array}$ & $\begin{array}{l}\text { Marked improve- } \\
\text { ment }\end{array}$ \\
\hline 18 & L. L. M. & $\mathrm{M}$ & 510,1937 & Acute rhinitis & Complete relief \\
\hline 19 & G. B. & $\mathrm{F}$ & $31,16,46,1937$ & Acute cold & Complete relief \\
\hline 20 & H. C. & $\mathrm{F}$ & $320,21,1937$ & $\begin{array}{l}\text { Bilateral maxillary } \\
\text { sinusicis }\end{array}$ & $\begin{array}{l}\text { Marked improve- } \\
\text { mene }\end{array}$ \\
\hline 21 & R. M. & $\mathrm{F}$ & 321,1937 & Acute cold & Complete relicf \\
\hline
\end{tabular}


TABLE II-(Continued)

\begin{tabular}{|c|c|c|c|c|c|}
\hline Case \# & Patient & $\operatorname{Sex}$ & Date & Diagnosis & Comment \\
\hline 22 & J. O'D. & $\mathbf{M}$ & 320,1937 & Acute cold & Complese relief \\
\hline 23 & J. N. & M & $323,24,31,1937$ & $\begin{array}{l}\text { Bilateral maxillary } \\
\text { sinusitis }\end{array}$ & $\begin{array}{l}\text { Marked improve- } \\
\text { ment }\end{array}$ \\
\hline 24 & E. S. & $F$ & 325,1937 & Acuze cold & Complete relief \\
\hline 25 & R. G. & $\mathrm{F}$ & 328,1937 & Acute cold & Complete relief \\
\hline 26 & A.S. & $\mathbf{M}$ & $\begin{array}{l}1021,25,1113 \\
1221,1936 ; 1 \% 15 \\
29,224,25,1937\end{array}$ & $\begin{array}{l}\text { Acute rhinitis; } \\
\text { deflected septum, } \\
\text { ethmoid, poly- } \\
\text { poid }\end{array}$ & $\begin{array}{l}\text { Marked improve- } \\
\text { ment }\end{array}$ \\
\hline 27 & C. R. & $\mathrm{F}$ & $10^{\prime} 22,11: 12,1936$ & Acute rhinitis & $\begin{array}{l}\text { Completely } \mathrm{re}^{2}- \\
\text { lieved }\end{array}$ \\
\hline 28 & H. R. & $\mathbf{M}$ & $\begin{array}{l}10,23,26,11 ; 27 \\
30,1936 ; 1 ; 8,18 \\
22,29,2224,1937\end{array}$ & $\begin{array}{l}\text { Acute rhinicis } \\
\text { Bifateral maxillary } \\
\text { ethmoidal sinu- } \\
\text { sitis }\end{array}$ & $\begin{array}{l}\text { Acute symptoms } \\
\text { relieved; sinusi- } \\
\text { tis imptoved }\end{array}$ \\
\hline 29 & N. K. & $\mathrm{F}$ & $\begin{array}{c}1030,112,4,6 \\
11,1219,1936\end{array}$ & $\begin{array}{l}\text { Vasomotor rhin- } \\
\text { itis, severe }\end{array}$ & $\begin{array}{l}\text { Marked improve- } \\
\text { ment }\end{array}$ \\
\hline 30 & E. J. & $F$ & $\begin{array}{l}11.2,9,13,17 \\
1936\end{array}$ & $\begin{array}{l}\text { Bilateral ethmoid- } \\
\text { ícis }\end{array}$ & $\begin{array}{l}\text { Marked improve- } \\
\text { ment }\end{array}$ \\
\hline 31 & S. W'. & $\mathrm{F}$ & 112.1936 & Acute cold & Complete relief́ \\
\hline 32 & W. J.R. & $\mathrm{M}$ & $113,9,23,1936$ & $\begin{array}{l}\text { Acute rhinitis } \\
\text { Bilateral ethmoid- } \\
\text { itis }\end{array}$ & $\begin{array}{l}\text { Marked improve- } \\
\text { ment }\end{array}$ \\
\hline 33 & A. S. & $\mathrm{F}$ & $113,9,11,14,1936$ & $\begin{array}{l}\text { Deflected seprum } \\
\text { Bilateral ethmoid- } \\
\text { itis }\end{array}$ & $\begin{array}{l}\text { Marked improve- } \\
\text { ment }\end{array}$ \\
\hline 34 & S. T. & $\mathrm{F}$ & $\begin{array}{l}10 \cdot 29,113,10,25 \\
1936\end{array}$ & $\begin{array}{l}\text { Bilateral erhmoid- } \\
\text { itis; spheno-pal- } \\
\text { atine ganglion } \\
\text { neuralgia }\end{array}$ & $\begin{array}{l}\text { Marked improve- } \\
\text { ment }\end{array}$ \\
\hline 35 & G. F. & M & 114,1936 & Acute cold & Complete relief \\
\hline 36 & A. G. & $\mathbf{M}$ & 114,1936 & Acute rhinitis & Complere relief $f$ \\
\hline 37 & S. R. & $M$ & 1016,1936 & Acute rhinitis & $\begin{array}{l}\text { Relieved con- } \\
\text { plesely }\end{array}$ \\
\hline 38 & S. H. & M & $\begin{array}{l}10115,23,26,1936 \\
18,310,12,1937\end{array}$ & $\begin{array}{l}\text { Acute rhinitis } \\
\text { Bilateral ethmoid- } \\
\text { itis }\end{array}$ & $\begin{array}{l}\text { Relicved cum- } \\
\text { pletely }\end{array}$ \\
\hline
\end{tabular}


TABLl: $11-$ (Continued)

\begin{tabular}{|c|c|c|c|c|c|}
\hline Cust \# & l'ationt & Sex & Datc & Diagnnoxis & Camment \\
\hline 39 & F. L. & $M$ & 1020,1936 & $\begin{array}{l}\text { Acure rhinitis } \\
\text { left ethmoiditis }\end{array}$ & $\begin{array}{l}\text { Marked improve- } \\
\text { inent }\end{array}$ \\
\hline 40 & T. C. & $\mathrm{F}$ & $\begin{array}{l}1020,23,27,11 / 4 \\
17,22,9,14,21 \\
1936 ; 1,5,8,1937\end{array}$ & $\begin{array}{l}\text { Bilateral cthmoid- } \\
\text { itis }\end{array}$ & $\begin{array}{l}\text { Marked improwe- } \\
\text { ment }\end{array}$ \\
\hline+1 & L. A. & 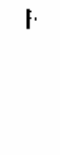 & $\begin{array}{l}1020,23,26,11 / 7 \\
1211,1936 ; 2 / 27 \\
1937\end{array}$ & $\begin{array}{l}\text { Acute rhinitis, de- } \\
\text { viated septum. } \\
\text { bilateral eth- } \\
\text { moiditis }\end{array}$ & $\begin{array}{l}\text { Relieved of acure } \\
\text { atracks. Sinusitis } \\
\text { improved }\end{array}$ \\
\hline+2 & F. R. & $F$ & $1010,21,1936$ & Acutc rhinitis & $\begin{array}{l}\text { Relieved cons- } \\
\text { plctely }\end{array}$ \\
\hline+3 & A. C. & $M$ & $\begin{array}{l}1021,24,26,11 / 6, \\
9,11,12,1936\end{array}$ & $\begin{array}{l}\text { Acute rhinitis } \\
\text { Bilateral ethmaid. } \\
\text { itis }\end{array}$ & $\begin{array}{l}\text { Marked improve- } \\
\text { ment }\end{array}$ \\
\hline 44 & F.S. & $\mathbf{M}$ & $\begin{array}{c}10 / 21,25,28,12 / 14 \\
1936 ; 1: 27,3 / 10 \\
12,47,1937\end{array}$ & $\begin{array}{l}\text { Acute rhinitis, } \\
\text { left pan-sinusitis: } \\
\text { left radical pan- } \\
\text { sinus operation t } \\
\text { yrs. ago }\end{array}$ & $\begin{array}{l}\text { Narked improwe- } \\
\text { ment }\end{array}$ \\
\hline+5 & J. E. & $\mathrm{M}$ & 1021,1936 & Acute rhinitis & $\begin{array}{l}\text { Relieved com- } \\
\text { pletely }\end{array}$ \\
\hline+6 & E. E. & $\mathrm{F}$ & $\begin{array}{l}1021,28,11: 4, \\
1936\end{array}$ & $\begin{array}{l}\text { Acute rhinitis } \\
\text { Bilateral ethmoid- } \\
\text { itis }\end{array}$ & $\begin{array}{l}\text { Completely re- } \\
\text { lieved }\end{array}$ \\
\hline 47 & L. C. & $\mathrm{I}:$ & $\begin{array}{l}1022,26,29,1123 \\
27,1936\end{array}$ & $\begin{array}{l}\text { Acute rhinitis } \\
\text { liilateral ethmeid- } \\
\text { itis }\end{array}$ & $\begin{array}{l}\text { Relieved con- } \\
\text { pletely }\end{array}$ \\
\hline 48 & J. W. & $M$ & $119,12,1936$ & Acute shinitis & $\begin{array}{l}\text { Marked improve- } \\
\text { ment }\end{array}$ \\
\hline+9 & A. F. & $\mathrm{F}$ & $\begin{array}{l}1023,26,1117,23 \\
1936\end{array}$ & $\begin{array}{l}\text { Acute thinitis: } \\
\text { nasal allerky }\end{array}$ & $\begin{array}{l}\text { Marked injprove- } \\
\text { ment }\end{array}$ \\
\hline s0 & A. K. & $F$ & 1026,1936 & $\begin{array}{l}\text { Acute rhinitis } \\
\text { Bilateral ethmoid- } \\
\text { itis; left sphe- } \\
\text { noiditis }\end{array}$ & Marked relief \\
\hline$s$ & B. S. & ; & 1026,1936 & Acute rhinitis & $\begin{array}{l}\text { Completely re- } \\
\text { lieved }\end{array}$ \\
\hline$\$ 2$ & L. S. & F & 1026,1936 & $\begin{array}{l}\text { Acute rhinitis: } \\
\text { right maxillary } \\
\text { sinusitis, old } \\
\text { radical operation }\end{array}$ & $\begin{array}{l}\text { Marked improve- } \\
\text { ment }\end{array}$ \\
\hline
\end{tabular}


TABLE $1 /-$ (Continued)

\begin{tabular}{|c|c|c|c|c|c|}
\hline Case \# & Patient & $\operatorname{Sex}$ & Date & Diagnosis & Comment \\
\hline 73 & G. N. & $M$ & 424,1937 & $\begin{array}{l}\text { Bilnteral ethmoid- } \\
\text { itis }\end{array}$ & Complese relief \\
\hline 74 & E. H. & F & $426,29,19.37$ & Acute cold & Complete' relief \\
\hline 75 & J.F. & $M$ & 818,1937 & Allergic rhinitis & $\begin{array}{l}\text { Marked improve- } \\
\text { ment }\end{array}$ \\
\hline 76 & A. B. & $\mathrm{F}$ & $8: 18,1937$ & $\begin{array}{l}\text { Acute otitis, } \\
\text { grippe type } \\
\text { Acute rhinitis }\end{array}$ & $\begin{array}{c}\text { Improved. Xo } \\
\text { parncentesis }\end{array}$ \\
\hline 77 & M. R. & $\mathbf{F}$ & $6: 25,28,1937$ & Acute rhinitis & Complete relief \\
\hline 78 & J. B. & $\mathbf{M}$ & 1028,1937 & Acute told & Complets relief \\
\hline 79 & A. 5 . & $\mathrm{M}$ & $78,13,816,1937$ & $\begin{array}{l}\text { Nasal allerty; } \\
\text { Bilat. maxillary } \\
\text { and exhmoidal } \\
\text { sinusitis }\end{array}$ & $\begin{array}{l}\text { Marked improve- } \\
\text { ment }\end{array}$ \\
\hline 80 & B. L. & $\mathrm{F}$ & $7,12,16,1937$ & $\begin{array}{l}\text { Acute rhinitis and } \\
\text { bronchitis }\end{array}$ & $\begin{array}{l}\text { Marked improve- } \\
\text { ment }\end{array}$ \\
\hline 81 & I. 1. & $\mathrm{~F}$ & $\begin{array}{l}717,89,25,916 \\
20,24,27,10 \cdot 1,3 \\
7,15,18,19,1937\end{array}$ & $\begin{array}{l}\text { Bilateral ethmoid- } \\
\text { itis }\end{array}$ & $\begin{array}{l}\text { Moderately ini- } \\
\text { preved }\end{array}$ \\
\hline 82 & L. $S$. & F & $\begin{array}{l}717,23,84,6,13 \\
20,98,12,28 \\
1937\end{array}$ & $\begin{array}{l}\text { Bilateral echmoid- } \\
\text { itis }\end{array}$ & $\begin{array}{l}\text { Marked improve- } \\
\text { ment }\end{array}$ \\
\hline 83 & L. I. & $M$ & $4 / 9,1937$ & Allergic rhinitis & $\begin{array}{l}\text { Marked improve- } \\
\text { ment }\end{array}$ \\
\hline 84 & A. C. & $\mathrm{F}$ & 410,1937 & $\begin{array}{l}\text { Bilateral ethmoid- } \\
\text { itis }\end{array}$ & $\begin{array}{l}\text { Marked impruve- } \\
\text { ment }\end{array}$ \\
\hline 85 & S. F. & $M$ & $41,19,1937$ & $\begin{array}{l}\text { Acute cold, post- } \\
\text { nasal drip }\end{array}$ & $\begin{array}{l}\text { Marked inlpruve- } \\
\text { ment }\end{array}$ \\
\hline 86 & R. L. & $\mathrm{F}$ & $45,17,1937$ & $\begin{array}{l}\text { Bilateral ethmoid- } \\
\text { itis. Sinusitis }\end{array}$ & $\begin{array}{l}\text { Moderately im- } \\
\text { proved }\end{array}$ \\
\hline 87 & F. W. & $\mathrm{F}$ & 117,1936 & Acute cold & Complete relicf \\
\hline 88 & L. $S$. & M & $117,9,1936$ & Acute cold & Complete relief \\
\hline 89 & M. R. & $\mathrm{M}$ & 117,1936 & Acute cold & Completi relicf \\
\hline 90 & O. F. & $\mathrm{M}$ & 1028,1036 & Acute rhinitis & Complete relief \\
\hline 91 & I. B. & $\mathrm{F}$ & $10 \cdot 28,116,16,1936$ & $\begin{array}{l}\text { Deviated seprum, } \\
\text { Bilateral exhmoid- } \\
\text { itis; acute rhin- } \\
\text { itis }\end{array}$ & $\begin{array}{l}\text { Marked improve- } \\
\text { ment }\end{array}$ \\
\hline
\end{tabular}


TABLE It-(Continued)

\begin{tabular}{|c|c|c|c|c|c|}
\hline Case \# & Pationt & Sex & Date & Diagnosis & Comment \\
\hline 92 & R. T. & $\mathrm{Ni}$ & $1028,112,16,1936$ & $\begin{array}{l}\text { Sinusitis; bilacera] } \\
\text { maxillary sinusi- } \\
\text { cis. Asthma }\end{array}$ & $\begin{array}{l}\text { Marked improve- } \\
\text { ment in asthma } \\
\text { and sinusitis }\end{array}$ \\
\hline 93 & C. N. & $M$ & 1030,1936 & Acute rhinitis & $\begin{array}{l}\text { Completely re- } \\
\text { licved }\end{array}$ \\
\hline 94 & C. A. & $\mathrm{M}$ & 1030.1936 & Acute rhinitis & $\begin{array}{l}\text { Completely rt- } \\
\text { lieved }\end{array}$ \\
\hline 95 & A. M. & $\mathbf{M}$ & $10.31,11,7,21,1936$ & $\begin{array}{l}\text { Allergic vasomo- } \\
\text { tor rhinitis }\end{array}$ & $\begin{array}{l}\text { Marked improve- } \\
\text { ment }\end{array}$ \\
\hline 96 & R. M. & $F$ & $\begin{array}{l}111,9,19,122 \\
20,1936: 1.5,10 \\
15,219,25,316 \\
1937\end{array}$ & $\begin{array}{l}\text { Post-nasal drip } \\
\text { Bilateral ethmiod- } \\
\text { itis }\end{array}$ & $\begin{array}{l}\text { Marked improve- } \\
\text { ment }\end{array}$ \\
\hline-97 & D. $\mathrm{K}$. & $\mathrm{F}$ & $\begin{array}{l}10: 14,20,11 / 2,9 \\
1936\end{array}$ & $\begin{array}{l}\text { Altergic rhinitis. } \\
\text { Polypoid pan-sin- } \\
\text { usitis. Deflected } \\
\text { septum }\end{array}$ & $\begin{array}{l}\text { Allergy improved } \\
\text { greatly. Sinusitis } \\
\text { recurred. Radi- } \\
\text { cal operation }\end{array}$ \\
\hline 98 & N.G. & $\mathrm{F}$ & $\begin{array}{l}716,927,1025 \\
1937\end{array}$ & $\begin{array}{l}\text { Bilateral maxillary } \\
\text { sinusitis. Severe } \\
\text { asthma, nasal al- } \\
\text { lergy }\end{array}$ & $\begin{array}{l}\text { Asthma complete- } \\
\text { ly relieved. Sinu- } \\
\text { sitis markedly } \\
\text { improved }\end{array}$ \\
\hline 99 & J. 5 . & $\mathrm{F}$ & $\begin{array}{l}628,714,1020 \\
23,1937\end{array}$ & $\begin{array}{l}\text { Bilateral maxillary } \\
\text { ethmoid sinusitis. } \\
\text { severe asthma, } \\
\text { nasal altergy }\end{array}$ & $\begin{array}{l}\text { Asthma narkedly } \\
\text { improved. Sinu- } \\
\text { sitis marksoly } \\
\text { improved }\end{array}$ \\
\hline 100 & J. G. & $\mathrm{M}$ & $\begin{array}{l}81,21,24,31,91 \\
3,16,25,102,9 \\
16,25,30,1937\end{array}$ & $\begin{array}{l}\text { Bilater.ul ethmoid- } \\
\text { iris. Nasal aller- } \\
\text { gy. Asthma }\end{array}$ & $\begin{array}{l}\text { Asthma complest- } \\
\text { ly relicved. Nasa] } \\
\text { allergy relieved. } \\
\text { Sinusitis marked- } \\
\text { lv improved. }\end{array}$ \\
\hline
\end{tabular}

\section{BIBLIOGRAPHY}

1. Cantarow, Abraham: Calcium Metabolism and Calcium Therapy. Lea \& Febinger, 1933.

2. Hastings, A. B., Murray, C. D., and Sendray, J., Jr.: Journal Biological Chemistry, 71:723, 1926.

3. Greenwald, I.: Journal Biological Chemistry, 67:1, 1926; Journal Biological Chemistry, 93:551, 1931.

4. Klinke, K.: Klin. Wochschr., 6:791, 1927. 


\section{Ruskin 1938}

This text was scanned and changed to characters using an Optical Character Recognition program. The program may generate a few errors, but they have been corrected, although few may remain.

This file (version Nov 23, 2007):

http://www.ltdk.helsinki.fi/users/hemila/pauling/Ruskin_1938.pdf

\section{Harri Hemilä}

Department of Public Health

University of Helsinki, Helsinki, Finland

harri.hemila@helsinki.fi

Home: http://www.ltdk.helsinki.fi/users/hemila 